\title{
Weak Dynamic Programming Principle for Viscosity Solutions *
}

\author{
Bruno Bouchard ${ }^{\dagger}$ and Nizar Touzi ${ }^{\ddagger}$
}

February 2009

\begin{abstract}
We prove a weak version of the dynamic programming principle for standard stochastic control problems and mixed control-stopping problems, which avoids the technical difficulties related to the measurable selection argument. In the Markov case, our result is tailor-maid for the derivation of the dynamic programming equation in the sense of viscosity solutions.
\end{abstract}

Key words: Optimal control, Dynamic programming, discontinuous viscosity solutions.

AMS 1991 subject classifications: Primary 49L25, 60J60; secondary 49L20, 35K55.

\section{Introduction}

Consider the standard class of stochastic control problems in the Mayer form

$$
V(t, x):=\sup _{\nu \in \mathcal{U}} \mathbb{E}\left[f\left(X_{T}^{\nu}\right) \mid X_{t}^{\nu}=x\right]
$$

where $\mathcal{U}$ is the controls set, $X^{\nu}$ is the controlled process, $f$ is some given function, $0<T \leq \infty$ is a given time horizon, $t \in[0, T)$ is the time origin, and $x \in \mathbb{R}^{d}$ is some given initial condition. This framework includes the general class of stochastic control problems under the so-called Bolza formulation, the corresponding singular versions, and optimal stopping problems.

${ }^{*}$ The authors are grateful to Nicole El Karoui for fruitful comments. This research is part of the Chair Financial Risks of the Risk Foundation sponsored by Société Générale, the Chair Derivatives of the Future sponsored by the Fédération Bancaire Française, the Chair Finance and Sustainable Development sponsored by EDF and Calyon, and the Chair Les particuliers face au risque sponsored by Groupama.

†CEREMADE, Université Paris Dauphine and CREST-ENSAE, bouchard@ceremade.dauphine.fr

${ }^{\ddagger}$ Ecole Polytechnique Paris, Centre de Mathématiques Appliquées, touzi@cmap.polytechnique.fr 
A key-tool for the analysis of such problems is the so-called dynamic programming principle (DPP), which relates the time- $t$ value function $V(t,$.$) to any later time- \tau$ value $V(\tau,$.$) for$ any stopping time $\tau \in[t, T)$ a.s. A formal statement of the DPP is:

$$
{ }^{\prime \prime} V(t, x)=v(t, x):=\sup _{\nu \in \mathcal{U}} \mathbb{E}\left[V\left(\tau, X_{\tau}^{\nu}\right) \mid X_{t}^{\nu}=x\right] . "
$$

In particular, this result is routinely used in the case of controlled Markov jump-diffusions in order to derive the corresponding dynamic programming equation in the sense of viscosity solutions, see Lions [6, 7], Fleming and Soner [5], and Touzi [9].

The statement (1.1) of the DPP is very intuitive and can be easily proved in the deterministic framework, or in discrete-time with finite probability space. However, its proof is in general not trivial, and requires on the first stage that $V$ be measurable.

The inequality " $V \leq v$ " is the easy one but still requires that $V$ be measurable. Our weak formulation avoids this issue. Namely, under fairly general conditions on the controls set and the controlled process, it follows from an easy application of the tower property of conditional expectations that

$$
V(t, x) \leq \sup _{\nu \in \mathcal{U}} \mathbb{E}\left[V^{*}\left(\tau, X_{\tau}^{\nu}\right) \mid X_{t}^{\nu}=x\right]
$$

where $V^{*}$ is the upper semicontinuous envelope of the function $V$.

The proof of the converse inequality " $V \geq v$ " in a general probability space turns out to be difficult when the function $V$ is not known a priori to satisfy some continuity condition. See e.g. Bertsekas and Shreve [1], Borkar [2], and El Karoui [4].

Our weak version of the DPP avoids the non-trivial measurable selection argument needed to prove the inequality $V \geq v$ in (1.1). Namely, in the context of a general control problem presented in Section 2, we show in Section 3 that:

$$
V(t, x) \geq \sup _{\nu \in \mathcal{U}} \mathbb{E}\left[\varphi\left(\tau, X_{\tau}^{\nu}\right) \mid X_{t}=x\right]
$$

for every upper-semicontinuous minorant $\varphi$ of $V$.

We also show that an easy consequence of this result is that

$$
V(t, x) \geq \sup _{\nu \in \mathcal{U}} \mathbb{E}\left[V_{*}\left(\tau_{n}^{\nu}, X_{\tau_{n}^{\nu}}^{\nu}\right) \mid X_{t}=x\right]
$$

where $\tau_{n}^{\nu}:=\tau \wedge \inf \left\{s>t:\left|X_{s}^{\nu}-x\right|>n\right\}$, and $V_{*}$ is the lower semicontinuous envelope of $V$.

This result is weaker than the classical DPP (1.1). However, in the controlled Markov jumpdiffusions case, it turns out to be tailor-maid for the derivation of the dynamic programming equation in the sense of viscosity solutions. Section 5 reports this derivation in the context of controlled diffusions.

Finally, Section 4 provides an extension of our argument in order to obtain a weak dynamic programming principle for mixed control-stopping problems. 


\section{The stochastic control problem}

Let $(\Omega, \mathcal{F}, P)$ be a probability space, $T>0$ a finite time horizon, and $\mathbb{F}:=\left\{\mathcal{F}_{t}, 0 \leq t \leq T\right\}$ a given filtration of $\mathcal{F}$, satisfying the usual assumptions. For every $t \geq 0$, we denote by $\mathbb{F}^{t}=\left(\mathcal{F}_{s}^{t}\right)_{s \geq 0}$ the right-continuous filtration generated by $\mathbb{F}$ measurable processes that are independent of $\mathcal{F}_{t}, t \geq 0$.

We denote by $\mathcal{T}$ the collection of all $\mathbb{F}$-stopping times. For $\tau_{1}, \tau_{2} \in \mathcal{T}$ with $\tau_{1} \leq \tau_{2}$ a.s., the subset $\mathcal{T}_{\left[\tau_{1}, \tau_{2}\right]}$ is the collection of all $\tau \in \mathcal{T}$ such that $\tau \in\left[\tau_{1}, \tau_{2}\right]$ a.s. When $\tau_{1}=0$, we simply write $\mathcal{T}_{\tau_{2}}$. We use the notations $\mathcal{T}_{\left[\tau_{1}, \tau_{2}\right]}^{t}$ and $\mathcal{T}_{\tau_{2}}^{t}$ to denote the corresponding sets of stopping times that are independent of $\mathcal{F}_{t}$

For $\tau \in \mathcal{T}$ and a subset $A$ of a finite dimensional space, we denote by $\mathbb{L}_{\tau}^{0}(A)$ the collection of all $\mathcal{F}_{\tau}$-measurable random variables with values in $A . \mathbb{H}^{0}(A)$ is the collection of all $\mathbb{F}$-progressively measurable processes with values in $A$, and $\mathbb{H}_{\mathrm{rcll}}^{0}(A)$ is the subset of all processes in $\mathbb{H}^{0}(A)$ which are right-continuous with finite left limits.

In the following, we denote by $B_{r}(z)$ (resp. $\left.\partial B_{r}(z)\right)$ the open ball (resp. its boundary) of radius $r>0$ and center $z \in \mathbb{R}^{\ell}, \ell \in \mathbb{N}$.

Througout this note, we fix an integer $d \in \mathbb{N}$, and we introduce the sets:

$$
\mathbf{S}:=[0, T] \times \mathbb{R}^{d} \quad \text { and } \quad \mathcal{S}_{0}:=\left\{(\tau, \xi): \tau \in \mathcal{T}_{T} \text { and } \xi \in \mathbb{L}_{\tau}^{0}\left(\mathbb{R}^{d}\right)\right\} .
$$

We also denote by $\operatorname{USC}(\mathbf{S})$ (resp. $\operatorname{LSC}(\mathbf{S})$ ) the collection of all upper-semicontinuous (resp. lower-semicontinuous) functions from $\mathbf{S}$ to $\mathbb{R}$.

The set of control processes is a given subset $\mathcal{U}_{0}$ of $\mathbb{H}^{0}\left(\mathbb{R}^{k}\right)$, for some integer $k \geq 1$, so that the controlled state process defined as the mapping:

$$
(\tau, \xi ; \nu) \in \mathcal{S} \times \mathcal{U}_{0} \longmapsto X_{\tau, \xi}^{\nu} \in \mathbb{H}_{\text {rcll }}^{0}\left(\mathbb{R}^{d}\right) \text { for some } \mathcal{S} \text { with } \mathbf{S} \subset \mathcal{S} \subset \mathcal{S}_{0}
$$

is well-defined and satisfies:

$$
\left(\theta, X_{\tau, \xi}^{\nu}(\theta)\right) \in \mathcal{S} \text { for all }(\tau, \xi) \in \mathcal{S} \text { and } \theta \in \mathcal{T}_{[\tau, T]} .
$$

Given a Borel function $f: \mathbb{R}^{d} \longrightarrow \mathbb{R}$ and $(t, x) \in \mathbf{S}$, we introduce the reward function $J: \mathbf{S} \times \mathcal{U} \longrightarrow \mathbb{R}:$

$$
J(t, x ; \nu):=\mathbb{E}\left[f\left(X_{t, x}^{\nu}(T)\right)\right]
$$

which is well-defined for controls $\nu$ in

$$
\mathcal{U}:=\left\{\nu \in \mathcal{U}_{0}: \mathbb{E}\left|f\left(X_{t, x}^{\nu}(T)\right)\right|<\infty \forall(t, x) \in \mathbf{S}\right\} .
$$

We say that a control $\nu \in \mathcal{U}$ is $t$-admissible if it is independent of $\mathcal{F}_{t}$, and we denote by $\mathcal{U}_{t}$ the collection of such processes. The stochastic control problem is defined by:

$$
V(t, x):=\sup _{\nu \in \mathcal{U}_{t}} J(t, x ; \nu) \quad \text { for } \quad(t, x) \in \mathbf{S} .
$$




\section{Dynamic programming for stochastic control prob- lems}

For the purpose of our weak dynamic programming principle, the following assumptions are crucial.

Assumption A For all $(t, x) \in \mathbf{S}$ and $\nu \in \mathcal{U}_{t}$, the controlled state process satisfies:

A1 (Independence) The process $X_{t, x}^{\nu}$ is independent of $\mathcal{F}_{t}$.

A2 (Causality) For $\tilde{\nu} \in \mathcal{U}_{t}$, if $\nu=\tilde{\nu}$ on $A \subset \mathcal{F}$, then $X_{t, x}^{\nu}=X_{t, x}^{\tilde{\nu}}$ on $A$.

A3 (Stability under concatenation) For every $\tilde{\nu} \in \mathcal{U}_{t}$, and $\theta \in \mathcal{T}_{[t, T]}^{t}$ :

$$
\nu \mathbf{1}_{[0, \theta]}+\tilde{\nu} \mathbf{1}_{(\theta, T]} \in \mathcal{U}_{t} .
$$

A4 (Consistency with deterministic initial data) For all $\theta \in \mathcal{T}_{[t, T]}^{t}$, we have:

a. For $\mathbb{P}$-a.e $\omega \in \Omega$, there exists $\tilde{\nu}_{\omega} \in \mathcal{U}_{\theta(\omega)}$ such that

$$
\mathbb{E}\left[f\left(X_{t, x}^{\nu}(T)\right) \mid \mathcal{F}_{\theta}\right](\omega) \leq J\left(\theta(\omega), X_{t, x}^{\nu}(\theta)(\omega) ; \tilde{\nu}_{\omega}\right)
$$

b. For $t \leq s \leq T, \theta \in \mathcal{T}_{[t, s]}^{t}, \tilde{\nu} \in \mathcal{U}_{s}$, and $\bar{\nu}:=\nu \mathbf{1}_{[0, \theta]}+\tilde{\nu} \mathbf{1}_{(\theta, T]}$, we have:

$$
\mathbb{E}\left[f\left(X_{t, x}^{\bar{\nu}}(T)\right) \mid \mathcal{F}_{\theta}\right](\omega)=J\left(\theta(\omega), X_{t, x}^{\nu}(\theta)(\omega) ; \tilde{\nu}\right) \text { for } \mathbb{P}-\text { a.e. } \omega \in \Omega
$$

Remark 3.1 Assumption A3 above implies the following property of the controls set which will be needed later:

A5 (Stability under bifurcation) For $\nu_{1}, \nu_{2} \in \mathcal{U}_{t}, \tau \in \mathcal{T}_{[t, T]}^{t}$ and $A \in \mathcal{F}_{\tau}^{t}$, we have:

$$
\bar{\nu}:=\nu_{1} \mathbf{1}_{[0, \tau]}+\left(\nu_{1} \mathbf{1}_{A}+\nu_{2} \mathbf{1}_{A^{c}}\right) \mathbf{1}_{(\tau, T]} \in \mathcal{U}_{t}
$$

To see this, observe that $\tau_{A}:=T \mathbf{1}_{A}+\tau \mathbf{1}_{A^{c}}$ is a stopping time in $\mathcal{T}_{[t, T]}^{t}$, and $\bar{\nu}=\nu_{1} \mathbf{1}_{\left[0, \tau_{A}\right)}+$ $\nu_{2} \mathbf{1}_{\left[\tau_{A}, T\right]}$ is the concatenation of $\nu_{1}$ and $\nu_{2}$ at the stopping time $\tau_{A}$.

Iterating the above property, we see that for $0 \leq t \leq s \leq T$ and $\tau \in \mathcal{T}_{[t, T]}^{t}$, we have the following extension: for a finite sequence $\left(\nu_{1}, \ldots, \nu_{n}\right)$ of control in $\mathcal{U}_{t}$ with $\nu_{i}=\nu_{1}$ on $[0, \tau)$, and for a partion $\left(A_{i}\right)_{1 \leq i \leq n}$ of $\Omega$ with $A_{i} \in \mathcal{F}_{\tau}^{t}$ for every $i \leq n$ :

$$
\bar{\nu}:=\nu_{1} \mathbf{1}_{[0, \tau)}+\mathbf{1}_{[\tau, T]} \sum_{i=1}^{n} \nu_{i} \mathbf{1}_{A_{i}} \in \mathcal{U}_{t} .
$$

Our main result is the following weak version of the dynamic programming principle which uses the following notation:

$$
V_{*}(t, x):=\liminf _{\left(t^{\prime}, x^{\prime}\right) \rightarrow(t, x)} V\left(t^{\prime}, x^{\prime}\right), \quad V^{*}(t, x):=\limsup _{\left(t^{\prime}, x^{\prime}\right) \rightarrow(t, x)} V\left(t^{\prime}, x^{\prime}\right), \quad(t, x) \in \mathbf{S} .
$$


Theorem 3.1 Let Assumptions A hold true. Then for every $(t, x) \in \mathbf{S}$, and for all family of stopping times $\left\{\theta^{\nu}, \nu \in \mathcal{U}_{t}\right\} \subset \mathcal{T}_{[t, T]}^{t}$

$$
V(t, x) \leq \sup _{\nu \in \mathcal{U}_{t}} \mathbb{E}\left[V^{*}\left(\theta^{\nu}, X_{t, x}^{\nu}\left(\theta^{\nu}\right)\right)\right]
$$

Assume further that $J(. ; \nu) \in \operatorname{LSC}(\mathbf{S})$ for every $\nu \in \mathcal{U}_{0}$. Then, for any function $\varphi: \mathbf{S} \longrightarrow \mathbb{R}$ :

$$
\varphi \in \operatorname{USC}(\mathbf{S}) \text { and } V \geq \varphi \Longrightarrow V(t, x) \geq \sup _{\nu \in \mathcal{U}_{t}^{\varphi}} \mathbb{E}\left[\varphi\left(\theta^{\nu}, X_{t, x}^{\nu}\left(\theta^{\nu}\right)\right)\right]
$$

where $\mathcal{U}_{t}^{\varphi}=\left\{\nu \in \mathcal{U}_{t}: \mathbb{E}\left[\varphi\left(\theta^{\nu}, X_{t, x}^{\nu}\left(\theta^{\nu}\right)\right)^{+}\right]<\infty\right.$ or $\left.\mathbb{E}\left[\varphi\left(\theta^{\nu}, X_{t, x}^{\nu}\left(\theta^{\nu}\right)\right)^{-}\right]<\infty\right\}$.

Before proceeding to the proof of this result, we report the following consequence.

Corollary 3.1 Let the conditions of Theorem 3.1 hold. For $(t, x) \in \mathbf{S}$, let $\left\{\theta^{\nu}, \nu \in \mathcal{U}_{t}\right\} \subset$ $\mathcal{T}_{[t, T]}^{t}$ be a family of stopping times such that $X_{t, x}^{\nu} \mathbf{1}_{\left[t, \theta^{\nu}\right]}$ is $\mathbb{L}^{\infty}$-bounded for all $\nu \in \mathcal{U}_{t}$. Then,

$$
\sup _{\nu \in \mathcal{U}_{t}} \mathbb{E}\left[V_{*}\left(\theta^{\nu}, X_{t, x}^{\nu}\left(\theta^{\nu}\right)\right)\right] \leq V(t, x) \leq \sup _{\nu \in \mathcal{U}_{t}} \mathbb{E}\left[V^{*}\left(\theta^{\nu}, X_{t, x}^{\nu}\left(\theta^{\nu}\right)\right)\right] .
$$

Proof The right-hand side inequality is already provided in Theorem 3.1. It follows from standard arguments, see e.g. Lemma 3.5 in [8], that we can find a sequence of continuous functions $\left(\varphi_{n}\right)_{n}$ such that $\varphi_{n} \leq V_{*} \leq V$ for all $n \geq 1$ and such that $\varphi_{n}$ converges pointwise to $V_{*}$ on $[0, T] \times B_{r}(0)$. Set $\phi_{N}:=\min _{n \geq N} \varphi_{n}$ for $N \geq 1$ and observe that the sequence $\left(\phi_{N}\right)_{N}$ is non-decreasing and converges pointwise to $V_{*}$ on $[0, T] \times B_{r}(0)$. Applying (3.2) of Theorem 3.1 and using the monotone convergence Theorem, we then obtain:

$$
V(t, x) \geq \lim _{N \rightarrow \infty} \mathbb{E}\left[\phi_{N}\left(\theta^{\nu}, X_{t, x}^{\nu}\left(\theta^{\nu}\right)\right)\right]=\mathbb{E}\left[V_{*}\left(\theta^{\nu}, X_{t, x}^{\nu}\left(\theta^{\nu}\right)\right)\right] .
$$

Proof of Theorem 3.1 1. Let $\nu \in \mathcal{U}_{t}$ be arbitrary and set $\theta:=\theta^{\nu}$. The first assertion is a direct consequence of Assumption A4-a. Indeed, it implies that, for $\mathbb{P}$-almost all $\omega \in \Omega$, there exists $\tilde{\nu}_{\omega} \in \mathcal{U}_{\theta(\omega)}$ such that

$$
\mathbb{E}\left[f\left(X_{t, x}^{\nu}(T)\right) \mid \mathcal{F}_{\theta}\right](\omega) \leq J\left(\theta(\omega), X_{t, x}^{\nu}(\theta)(\omega) ; \tilde{\nu}_{\omega}\right) .
$$

Since, by definition, $J\left(\theta(\omega), X_{t, x}^{\nu}(\theta)(\omega) ; \tilde{\nu}_{\omega}\right) \leq V^{*}\left(\theta(\omega), X_{t, x}^{\nu}(\theta)(\omega)\right)$, it follows from the tower property of conditional expectations that

$$
\mathbb{E}\left[f\left(X_{t, x}^{\nu}(T)\right)\right]=\mathbb{E}\left[\mathbb{E}\left[f\left(X_{t, x}^{\nu}(T)\right) \mid \mathcal{F}_{\theta}\right]\right] \leq \mathbb{E}\left[V^{*}\left(\theta, X_{t, x}^{\nu}(\theta)\right)\right] .
$$

2. Let $\left\{\left(t_{i}, x_{i}\right), i \geq 1\right\}:=\mathbb{Q}^{d+1} \cap \mathbf{S}$, and let $\varepsilon>0$ be given. Then there is a sequence $\left(\nu^{i, \varepsilon}\right)_{i \geq 1} \subset \mathcal{U}_{0}$ such that:

$$
\nu^{i, \varepsilon} \in \mathcal{U}_{t_{i}} \quad \text { and } \quad J\left(t_{i}, x_{i} ; \nu^{i, \varepsilon}\right) \geq V\left(t_{i}, x_{i}\right)-\varepsilon, \text { for every } i \geq 1 .
$$


By the lower-semicontinuity of $J\left(. ; \nu^{i, \varepsilon}\right)$, together with the upper-semicontinuity of $\varphi$, we may find a sequence $\left(r_{i}\right)_{i \geq 1}$ of positive scalars so that

$$
\varphi\left(t_{i}, x_{i}\right)-\varphi\left(t^{\prime}, x^{\prime}\right) \geq-\varepsilon \quad \text { and } \quad J\left(t_{i}, x_{i} ; \nu^{i, \varepsilon}\right)-J\left(t^{\prime}, x^{\prime} ; \nu^{i, \varepsilon}\right) \leq \varepsilon \quad \text { for } \quad\left(t^{\prime}, x^{\prime}\right) \in B_{i}, i \geq 1,
$$

where

$$
B_{i}:=\left\{\left(t^{\prime}, x^{\prime}\right) \in \mathbf{S}: t^{\prime} \in\left[t_{i}-r_{i}, t_{i}\right],\left|x^{\prime}-x_{i}\right| \leq r_{i}\right\}, \quad i \geq 1 .
$$

By (3.4) together with the fact that $V \geq \varphi$, this implies by :

$$
J\left(t^{\prime}, x^{\prime} ; \nu^{i, \varepsilon}\right) \geq J\left(t_{i}, x_{i} ; \nu^{i, \varepsilon}\right)-\varepsilon \geq V\left(t_{i}, x_{i}\right)-2 \varepsilon \geq \varphi\left(t_{i}, x_{i}\right)-2 \varepsilon \geq \varphi\left(t^{\prime}, x^{\prime}\right)-3 \varepsilon \text { on } B_{i} .
$$

Set $A_{1}:=B_{1}, C_{0}:=\emptyset$, and define the sequence

$$
A_{i+1}:=B_{i+1} \backslash C_{i} \text { where } C_{i}:=C_{i-1} \cup A_{i}, i \geq 1 .
$$

With this construction, it follows from (3.5) that the countable family $\left(A_{i}\right)_{i \geq 1}$ satisfies

$$
\cup_{i} A_{i}=\mathbf{S}, A_{i} \cap A_{j}=\emptyset \text { for } i \neq j, \text { and } J\left(t^{\prime}, x^{\prime} ; \nu^{i, \varepsilon}\right) \geq \varphi\left(t^{\prime}, x^{\prime}\right)-3 \varepsilon \text { on } A_{i} \subset B_{i} .
$$

4. We now prove (3.2). Set $A^{n}:=\cup_{i \leq n} A_{i}, n \geq 1$. Given $\nu \in \mathcal{U}_{t}$, we define

$$
\nu_{s}^{\varepsilon, n}:=\mathbf{1}_{[t, \theta]}(s) \nu_{s}+\mathbf{1}_{(\theta, T]}(s)\left(\nu_{s} \mathbf{1}_{\left(A^{n}\right)^{c}}\left(\theta, X_{t, x}^{\nu}(\theta)\right)+\sum_{i=1}^{n} \mathbf{1}_{A_{i}}\left(\theta, X_{t, x}^{\nu}(\theta)\right) \nu_{s}^{i, \varepsilon}\right), \quad \text { for } \quad s \in[t, T] .
$$

Notice that $\left\{\left(\theta, X_{t, x}^{\nu}(\theta)\right) \in A_{i}\right\} \in \mathcal{F}_{\theta}^{t}$ as a consequence of the independence Assumption A1. Then, it follows from the stability under concatenation Assumption A3 and Remark 3.1 that $\nu^{\varepsilon, n} \in \mathcal{U}_{t}$. Then, using Assumptions A4-b, A2, and (3.6), we deduce that:

$$
\begin{aligned}
\mathbb{E}\left[f\left(X_{t, x}^{\nu^{\varepsilon, n}}(T)\right) \mid \mathcal{F}_{\theta}\right] \mathbf{1}_{A^{n}}\left(\theta, X_{t, x}^{\nu}(\theta)\right) & =\sum_{i=1}^{n} J\left(\theta, X_{t, x}^{\nu}(\theta) ; \nu^{i, \varepsilon}\right) \mathbf{1}_{A_{i}}\left(\theta, X_{t, x}^{\nu}(\theta)\right) \\
& \geq \sum_{i=1}^{n}\left(\varphi\left(\theta, X_{t, x}^{\nu}(\theta)\right)-3 \varepsilon\right) \mathbf{1}_{A_{i}}\left(\theta, X_{t, x}^{\nu}(\theta)\right) \\
& =\left(\varphi\left(\theta, X_{t, x}^{\nu}(\theta)\right)-3 \varepsilon\right) \mathbf{1}_{A^{n}}\left(\theta, X_{t, x}^{\nu}(\theta)\right),
\end{aligned}
$$

which, by definition of $V$ and the tower property of conditional expectations, implies

$$
\begin{aligned}
V(t, x) & \geq J\left(t, x ; \nu^{\varepsilon, n}\right) \\
& =\mathbb{E}\left[\mathbb{E}\left[f\left(X_{t, x}^{\nu^{\varepsilon, n}}(T)\right) \mid \mathcal{F}_{\theta}\right]\right] \\
& \geq \mathbb{E}\left[\left(\varphi\left(\theta, X_{t, x}^{\nu}(\theta)\right)-3 \varepsilon\right) \mathbf{1}_{A^{n}}\left(\theta, X_{t, x}^{\nu}(\theta)\right)\right]+\mathbb{E}\left[f\left(X_{t, x}^{\nu}(T)\right) \mathbf{1}_{\left(A^{n}\right)^{c}}\left(\theta, X_{t, x}^{\nu}(\theta)\right)\right] .
\end{aligned}
$$

Since $f\left(X_{t, x}^{\nu}(T)\right) \in \mathbb{L}^{1}$, it follows from the dominated convergence theorem that:

$$
\begin{aligned}
V(t, x) \geq & -3 \varepsilon+\liminf _{n \rightarrow \infty} \mathbb{E}\left[\varphi\left(\theta, X_{t, x}^{\nu}(\theta)\right) \mathbf{1}_{A^{n}}\left(\theta, X_{t, x}^{\nu}(\theta)\right)\right] \\
= & -3 \varepsilon+\lim _{n \rightarrow \infty} \mathbb{E}\left[\varphi\left(\theta, X_{t, x}^{\nu}(\theta)\right)^{+} \mathbf{1}_{A^{n}}\left(\theta, X_{t, x}^{\nu}(\theta)\right)\right] \\
& \quad-\lim _{n \rightarrow \infty} \mathbb{E}\left[\varphi\left(\theta, X_{t, x}^{\nu}(\theta)\right)^{-} \mathbf{1}_{A^{n}}\left(\theta, X_{t, x}^{\nu}(\theta)\right)\right] \\
= & -3 \varepsilon+\mathbb{E}\left[\varphi\left(\theta, X_{t, x}^{\nu}(\theta)\right)\right],
\end{aligned}
$$


where the last equality follows from the monotone convergence theorem, due to the fact that either $\mathbb{E}\left[\varphi\left(\theta, X_{t, x}^{\nu}(\theta)\right)^{+}\right]<\infty$ or $\mathbb{E}\left[\varphi\left(\theta, X_{t, x}^{\nu}(\theta)\right)^{-}\right]<\infty$. The proof of (3.2) is completed by the arbitrariness of $\nu \in \mathcal{U}_{t}$ and $\varepsilon>0$.

Remark 3.2 (Lower-semicontinuity condition I) It is clear from the above proof that it suffices to prove the lower-semicontinuity of $(t, x) \mapsto J(t, x ; \nu)$ for $\nu$ in a subset $\tilde{\mathcal{U}}_{0}$ of $\mathcal{U}_{0}$ such that $\sup _{\nu \in \tilde{\mathcal{U}}_{t}} J(t, x ; \nu)=V(t, x)$. In most applications, this allows to reduce to the case where the controls are essentially bounded or satisfy a strong integrability condition.

Remark 3.3 (Lower-semicontinuity condition II) In the above proof, the lower-semicontinuity assumption is only used to construct the balls $B_{i}$ on which $J\left(t_{i}, x_{i} ; \nu^{i, \varepsilon}\right)-J\left(\cdot ; \nu^{i, \varepsilon}\right) \leq \varepsilon$. Clearly, it can be alleviated, and it suffices that the lower-semicontinuity holds in time from the left, i.e.

$$
\liminf _{\left(t^{\prime}, x^{\prime}\right) \rightarrow\left(t_{i}, x_{i}\right), t^{\prime} \leq t_{i}} J\left(t^{\prime}, x^{\prime} ; \nu^{i, \varepsilon}\right) \geq J\left(t_{i}, x_{i} ; \nu^{i, \varepsilon}\right) .
$$

Remark 3.4 (The Bolza formulation) Consider the stochastic control problem under the so-called Lagrange formulation:

$$
V(t, x):=\sup _{\nu \in \mathcal{U}_{t}} \mathbb{E}\left[\int_{t}^{T} Y_{t, x, 1}^{\nu}(s) g\left(s, X_{t, x}^{\nu}(s), \nu_{s}\right) d s+Y_{t, x, 1}^{\nu}(T) f\left(X_{t, x}^{\nu}(T)\right)\right],
$$

where

$$
d Y_{t, x, y}^{\nu}(s)=-Y_{t, x, y}^{\nu}(s) k\left(s, X_{t, x}^{\nu}(s), \nu_{s}\right) d s, Y_{t, x, y}^{\nu}(t)=y>0 .
$$

Then, it is well known that this problem can be converted into the Mayer formulation (2.3) by increasing the state process to $(X, Y, Z)$, where

$$
d Z_{t, x, y, z}^{\nu}(s)=Y_{t, x, y}^{\nu}(s) g\left(s, X_{t, x}^{\nu}(s), \nu_{s}\right) d s, Z_{t, x, y, z}^{\nu}(t)=z \in \mathbb{R},
$$

and considering the value function

$$
\bar{V}(t, x, y, z):=\sup _{\nu \in \mathcal{U}_{t}} \mathbb{E}\left[Z_{t, x, y, z}^{\nu}(T)+Y_{t, x, y}^{\nu}(T) f\left(X_{t, x}^{\nu}(T)\right)\right]=y V(t, x)+z .
$$

In particular, $V(t, x)=\bar{V}(t, x, 1,0)$. The first assertion of Theorem 3.1 implies

$$
V(t, x) \leq \sup _{\nu \in \mathcal{U}_{t}} \mathbb{E}\left[Y_{t, x, 1}^{\nu}\left(\theta^{\nu}\right) V\left(\theta^{\nu}, X_{t, x}^{\nu}\left(\theta^{\nu}\right)\right)+\int_{t}^{\theta^{\nu}} Y_{t, x, 1}^{\nu}(s) g\left(s, X_{t, x}^{\nu}(s), \nu_{s}\right) d s\right]
$$

Given a upper-semicontinuous minorant $\varphi$ of $V$, the function $\bar{\varphi}$ defined by $\bar{\varphi}(t, x, y, z):=$ $y \varphi(t, x)+z$ is an upper-semicontinuous minorant of $\bar{V}$. From the second assertion of Theorem 3.1 , we see that for a family $\left\{\theta^{\nu}, \nu \in \mathcal{U}_{t}\right\} \subset \mathcal{T}_{[t, T]}^{t}$,

$$
\begin{aligned}
V(t, x) & \geq \sup _{\nu \in \mathcal{U}_{t}^{\bar{\varphi}}} \mathbb{E}\left[\bar{\varphi}\left(\theta^{\nu}, X_{t, x}^{\nu}\left(\theta^{\nu}\right), Y_{t, x, 1}^{\nu}\left(\theta^{\nu}\right), Z_{t, x, 1,0}^{\nu}\left(\theta^{\nu}\right)\right)\right] \\
& =\sup _{\nu \in \mathcal{U}_{t}^{\bar{\varphi}}} \mathbb{E}\left[Y_{t, x, 1}^{\nu}\left(\theta^{\nu}\right) \varphi\left(\theta^{\nu}, X_{t, x}^{\nu}\left(\theta^{\nu}\right)\right)+\int_{t}^{\theta^{\nu}} Y_{t, x, 1}^{\nu}(s) g\left(s, X_{t, x}^{\nu}(s), \nu_{s}\right) d s\right] .
\end{aligned}
$$


Remark 3.5 (Infinite Horizon) Infinite horizon problems can be handled similarly. Following the notations of the previous Remark 3.4, we introduce the infinite horizon stochastic control problem:

$$
V^{\infty}(t, x):=\sup _{\nu \in \mathcal{U}_{t}} \mathbb{E}\left[\int_{t}^{\infty} Y_{t, x, 1}^{\nu}(s) g\left(s, X_{t, x}^{\nu}(s), \nu_{s}\right) d s\right] .
$$

Then, it is immediately seen that $V^{\infty}$ satisfies the weak dynamic programming principle (3.7)-(3.8).

\section{Dynamic programming for mixed control-stopping problems}

In this section, we provide a direct extension of the dynamics programming principle of Theorem 3.1 to the larger class of mixed control and stopping problems.

In the context of the previous section, we define for a Borel function $f: \mathbb{R}^{d} \longrightarrow \mathbb{R}$ and $(t, x) \in \mathbf{S}$ the reward $\bar{J}: \mathbf{S} \times \overline{\mathcal{U}} \times \mathcal{T}_{[t, T]} \longrightarrow \mathbb{R}:$

$$
\bar{J}(t, x ; \nu, \tau):=\mathbb{E}\left[f\left(X_{t, x}^{\nu}(\tau)\right)\right],
$$

which is well-defined for every control $\nu$ in

$$
\overline{\mathcal{U}}:=\left\{\nu \in \mathcal{U}_{0}: \mathbb{E}\left[\sup _{t \leq s \leq T}\left|f\left(X_{t, x}^{\nu}(s)\right)\right|\right]<\infty \forall(t, x) \in \mathbf{S}\right\} .
$$

The mixed control-stopping problem is defined by:

$$
\bar{V}(t, x):=\sup _{(\nu, \tau) \in \overline{\mathcal{U}}_{t} \times \mathcal{T}_{[t, T]}^{t}} \bar{J}(t, x ; \nu, \tau),
$$

where $\overline{\mathcal{U}}_{t}$ is the subset of elements of $\overline{\mathcal{U}}$ that are independent of $\mathcal{F}_{t}$.

The key ingredient for the proof of (4.6) is the following property of the set of stopping times $\mathcal{T}_{T}$ :

For all $\theta, \tau_{1} \in \mathcal{T}_{T}^{t}$ and $\tau_{2} \in \mathcal{T}_{[\theta, T]}^{t}$, we have $\tau_{1} \mathbf{1}_{\left\{\tau_{1}<\theta\right\}}+\tau_{2} \mathbf{1}_{\left\{\tau_{1} \geq \theta\right\}} \in \mathcal{T}_{T}^{t}$.

In order to extend the result of Theorem 3.1, we shall assume that the following version of A4 holds:

Assumption A4' For all $(t, x) \in \mathbf{S},(\nu, \tau) \in \overline{\mathcal{U}}_{t} \times \mathcal{T}_{[t, T]}^{t}$ and $\theta \in \mathcal{T}_{[t, T]}^{t}$, we have:

a. For $\mathbb{P}$-a.e $\omega \in \Omega$, there exists $\left(\tilde{\nu}_{\omega}, \tilde{\tau}_{\omega}\right) \in \overline{\mathcal{U}}_{\theta(\omega)} \times \mathcal{T}_{[\theta(\omega), T]}^{\theta(\omega)}$ such that

$$
\mathbb{E}\left[f\left(X_{t, x}^{\nu}(\tau)\right) \mid \mathcal{F}_{\theta}\right](\omega) \leq J\left(\theta(\omega), X_{t, x}^{\nu}(\theta)(\omega) ; \tilde{\nu}_{\omega}, \tilde{\tau}_{\omega}\right)
$$


b. For $t \leq s \leq T, \theta \in \mathcal{T}_{[t, s]}^{t}, \quad(\tilde{\nu}, \tilde{\tau}) \in \overline{\mathcal{U}}_{s} \times \mathcal{T}_{[s, T]}^{s}, \bar{\tau}:=\tau \mathbf{1}_{\{\tau<\theta\}}+\tilde{\tau} \mathbf{1}_{\{\tau \geq \theta\}}$, and $\bar{\nu}:=$ $\nu \mathbf{1}_{[0, \theta]}+\tilde{\nu} \mathbf{1}_{(\theta, T]}$, we have:

$$
\mathbb{E}\left[f\left(X_{t, x}^{\bar{\nu}}(\bar{\tau})\right) \mid \mathcal{F}_{\theta}\right](\omega)=J\left(\theta(\omega), X_{t, x}^{\nu}(\theta)(\omega) ; \tilde{\nu}, \tilde{\tau}\right) \quad \text { for } \mathbb{P}-\text { a.e. } \quad \omega \in \Omega .
$$

Theorem 4.1 Let Assumptions A1, A2, A3 and A4' hold true. Then for every $(t, x) \in \mathbf{S}$, and for all family of stopping times $\left\{\theta^{\nu}, \nu \in \overline{\mathcal{U}}_{t}\right\} \subset \mathcal{T}_{[t, T]}^{t}$ :

$$
\bar{V}(t, x) \leq \sup _{(\nu, \tau) \in \overline{\mathcal{U}}_{t} \times \mathcal{T}_{[t, T]}^{t}} \mathbb{E}\left[\mathbf{1}_{\left\{\tau<\theta^{\nu}\right\}} f\left(X_{t, x}^{\nu}(\tau)\right)+\mathbf{1}_{\left\{\tau \geq \theta^{\nu}\right\}} \bar{V}^{*}\left(\theta^{\nu}, X_{t, x}^{\nu}\left(\theta^{\nu}\right)\right)\right] .
$$

Assume further that the map $(t, x) \longmapsto \bar{J}(t, x ; \nu, \tau)$ satisfies the following lower-semicontinuity property

$$
\liminf _{t^{\prime} \uparrow t, x^{\prime} \rightarrow x} \bar{J}\left(t^{\prime}, x^{\prime} ; \nu, \tau\right) \geq \bar{J}(t, x ; \nu, \tau) \quad \text { for every } \quad(t, x) \in \mathbf{S} \text { and }(\nu, \tau) \in \overline{\mathcal{U}} \times \mathcal{T} .
$$

Then, for any function $\varphi \in \operatorname{USC}(\mathbf{S})$ with $\bar{V} \geq \varphi$ :

$$
\bar{V}(t, x) \geq \sup _{(\nu, \tau) \in \overline{\mathcal{U}}_{t}^{\varphi} \times \mathcal{T}_{[t, T]}^{t}} \mathbb{E}\left[\mathbf{1}_{\left\{\tau<\theta^{\nu}\right\}} f\left(X_{t, x}^{\nu}(\tau)\right)+\mathbf{1}_{\left\{\tau \geq \theta^{\nu}\right\}} \varphi\left(\theta^{\nu}, X_{t, x}^{\nu}\left(\theta^{\nu}\right)\right)\right],
$$

where $\overline{\mathcal{U}}_{t}^{\varphi}=\left\{\nu \in \overline{\mathcal{U}}_{t}: \mathbb{E}\left[\varphi\left(\theta^{\nu}, X_{t, x}^{\nu}\left(\theta^{\nu}\right)\right)^{+}\right]<\infty\right.$ or $\left.\mathbb{E}\left[\varphi\left(\theta^{\nu}, X_{t, x}^{\nu}\left(\theta^{\nu}\right)\right)^{-}\right]<\infty\right\}$.

For simplicity, we only provide the proof of Theorem 4.1 for optimal stopping problems, i.e. in the case where $\overline{\mathcal{U}}$ is reduced to a singleton. The dynamic programming principle for mixed control-stopping problems is easily proved by combining the arguments below with those of the proof of Theorem 3.1.

Proof (for optimal stopping problems) We omit the control $\nu$ from all notations, thus simply writing $X_{t, x}($.$) and \bar{J}(t, x ; \tau)$. Inequality (4.4) follows immediately from the tower property together with Assumptions A4'-a, recall that $\bar{J} \leq \bar{V}^{*}$.

We next prove (4.6). Arguying as in Step 2 of the proof of Theorem 3.1, we first observe that, for every $\varepsilon>0$, we can find a countable family $\bar{A}_{i}:=\left(t_{i}-r_{i}, t_{i}\right] \times A_{i} \subset \mathbf{S}$, together with a sequence of stopping times $\tau^{i, \varepsilon}$ in $\mathcal{T}_{\left[t_{i}, T\right]}^{t_{i}}, i \geq 1$, satisfying

$$
\cup_{i} \bar{A}_{i}=\mathbf{S}, \bar{A}_{i} \cap \bar{A}_{j}=\emptyset \text { for } i \neq j \text {, and } \bar{J}\left(\cdot ; \tau^{i, \varepsilon}\right) \geq \varphi-3 \varepsilon \text { on } \bar{A}_{i} .
$$

Set $\bar{A}^{n}:=\cup_{i \leq n} \bar{A}_{i}, n \geq 1$. Given two stopping times $\theta, \tau \in \mathcal{T}_{[t, T]}^{t}$, it follows from (4.3) and Assumption A1 that

$$
\tau^{n, \varepsilon}:=\tau \mathbf{1}_{\{\tau<\theta\}}+\mathbf{1}_{\{\tau \geq \theta\}}\left(T \mathbf{1}_{\left(\bar{A}^{n}\right)^{c}}\left(\theta, X_{t, x}(\theta)\right)+\sum_{i=1}^{n} \tau^{i, \varepsilon} \mathbf{1}_{\bar{A}_{i}}\left(\theta, X_{t, x}(\theta)\right)\right)
$$


is a stopping time in $\mathcal{T}_{[t, T]}^{t}$. We then deduce from the tower property together with Assumptions A4'-b and (4.7) that

$$
\begin{aligned}
\bar{V}(t, x) \geq & \bar{J}\left(t, x ; \tau^{n, \varepsilon}\right) \\
\geq & \mathbb{E}\left[f\left(X_{t, x}^{\nu}(\tau)\right) \mathbf{1}_{\{\tau<\theta\}}+\mathbf{1}_{\{\tau \geq \theta\}}\left(\varphi\left(\theta, X_{t, x}(\theta)\right)-3 \varepsilon\right) \mathbf{1}_{\bar{A}^{n}}\left(\theta, X_{t, x}(\theta)\right)\right] \\
& +\mathbb{E}\left[\mathbf{1}_{\{\tau \geq \theta\}} f\left(X_{t, x}(T)\right) \mathbf{1}_{\left(\bar{A}^{n}\right)^{c}}\left(\theta, X_{t, x}(\theta)\right)\right] .
\end{aligned}
$$

By sending $n \rightarrow \infty$ and arguing as in the end of the proof of Theorem 3.1, we deduce that

$$
\bar{V}(t, x) \geq \mathbb{E}\left[f\left(X_{t, x}(\tau)\right) \mathbf{1}_{\{\tau<\theta\}}+\mathbf{1}_{\{\tau \geq \theta\}} \varphi\left(\theta, X_{t, x}(\theta)\right)\right]-3 \varepsilon,
$$

and the result follows from the arbitrariness of $\varepsilon>0$ and $\tau \in \mathcal{T}_{[t, T]}$.

\section{Application to controlled diffusions}

In this section, we show how the weak DPP of Theorem 3.1 allows to derive the correponding dynamic programming equation in the sense of viscosity solutions. We refer, to Crandal, Ishii and Lions [3] and Fleming and Soner [5] for a presentation of the general theory of viscosity solutions.

For simplicity, we specialize the discussion to the context of controlled Markov diffusions driven by Brownian motions. Clearly the same technology can be adapted to optimal stopping, impulse control or mixed problems, and for controlled Markov jump-diffusions.

We take $(\Omega, \mathcal{F}, \mathbb{F}, \mathbb{P})$ to be the $d$-dimensional canonical filtered space equipped with the Wiener measure and denote by $\omega$ or $\tilde{\omega}$ a generic point. The Brownian motion is thus defined as $W(\omega)=\left(\omega_{t}\right)_{t \geq 0}$.

We let $U$ be a closed subset of $\mathbb{R}^{k}, k \geq 1$, and $\mu: \mathbf{S} \times U \longrightarrow \mathbb{R}^{d}$ and $\sigma: \mathbf{S} \times U \longrightarrow \mathbb{M}^{d}$ two Lipschitz continuous functions. Here $\mathbb{M}^{d}$ denotes the set of $d$-dimensional square matrices.

By $\mathcal{U}_{0}$, we denote the collection of all square integrable progressively measurable processes with values $U$. For every $\nu \in \mathcal{U}_{0}$, the stochastic differential equation:

$$
d X(r)=\mu\left(r, X(r), \nu_{r}\right) d r+\sigma\left(r, X(r), \nu_{r}\right) d W_{r}, \quad t \leq r \leq T
$$

has a unique strong solution $X_{\tau, \xi}^{\nu}$ satisfying $X_{\tau, \xi}^{\nu}(\tau)=\xi$, for any initial condition $(\tau, \xi) \in$ $\mathcal{S}:=\left\{(\tau, \xi) \in \mathcal{S}_{0}: \mathbb{E}\left[|\xi|^{2}\right]<\infty\right\}$, satisfying

$$
\mathbb{E}\left[\sup _{\tau \leq r \leq T}\left|X_{t, x}^{\nu}\right|^{2}\right]<\infty .
$$

The performance criterion $J$, and the value function $V$ are defined by (2.1)-(2.3) for a Borel function $f$ with quadratic growth. It follows that $\mathcal{U}=\mathcal{U}_{0}$ and the lower-semicontinuity assumption on $J$ follows from standard estimates. Moreover, it is easily checked that $V$ is locally bounded. 
Proposition 5.1 Under the above assumptions, the value function $V$ satisfies the weak dynamic programming principle (3.1)-(3.2).

Proof Conditions A1, A2 and A3 from Assumption A are obviously satisfied in the present context. It remains to check that A4 holds true. For $\omega \in \Omega$ and $r \geq 0$, we denote $\omega^{r}:=\omega_{\text {.^r }}$ and $\mathbf{T}_{r} \omega:=\omega_{.+r}-\omega_{r}$. Fix $(t, x) \in \mathbf{S}, \nu \in \mathcal{U}_{t}, \theta \in \mathcal{T}_{[t, T]}^{t}$, and observe that, by the flow property,

$$
\begin{aligned}
\mathbb{E}\left[f\left(X_{t, x}^{\nu}(T)\right) \mid \mathcal{F}_{\theta}\right](\omega) & =\int f\left(X_{\theta(\omega), X_{t, x}^{\nu}(\theta)(\omega)}^{\nu\left(\omega^{\theta(\omega)}+\mathbf{T}_{\theta(\omega)}(\omega)\right)}(T)\left(\mathbf{T}_{\theta(\omega)}(\omega)\right)\right) d \mathbb{P}\left(\mathbf{T}_{\theta(\omega)}(\omega)\right) \\
& =\int f\left(X_{\theta(\omega), X_{t, x}^{\nu}(\theta)(\omega)}^{\nu\left(\omega^{\theta(\omega)}+\mathbf{T}_{\theta(\omega)}(\tilde{\omega})\right)}(T)\left(\mathbf{T}_{\theta(\omega)}(\tilde{\omega})\right)\right) d \mathbb{P}(\tilde{\omega}) \\
& =J\left(\theta(\omega), X_{t, x}^{\nu}(\theta)(\omega) ; \tilde{\nu}_{\omega}\right)
\end{aligned}
$$

where, $\tilde{\nu}_{\omega}(\tilde{\omega}):=\nu\left(\omega^{\theta(\omega)}+\mathbf{T}_{\theta(\omega)} \tilde{\omega}\right)$ is an element of $\mathcal{U}_{\theta(\omega)}$. This already proves A4-a. As for A4-b, note that if $\bar{\nu}:=\nu \mathbf{1}_{[0, \theta]}+\tilde{\nu} \mathbf{1}_{(\theta, T]}$ with $\tilde{\nu} \in \mathcal{U}_{s}$ and $\theta \in \mathcal{T}_{[t, s]}^{t}$, then the same computations imply

$$
\mathbb{E}\left[f\left(X_{t, x}^{\bar{\nu}}(T)\right) \mid \mathcal{F}_{\theta}\right](\omega)=\int f\left(X_{\theta(\omega), X_{t, x}^{\nu}(\theta)(\omega)}^{\tilde{\nu}\left(\omega^{\theta(\omega)}+\mathbf{T}_{\theta(\omega)} \tilde{\omega}\right)}(T)\left(\mathbf{T}_{\theta(\omega)} \tilde{\omega}\right)\right) d \mathbb{P}(\tilde{\omega}),
$$

where we used the flow property together with the fact that $X_{t, x}^{\nu}=X_{t, x}^{\bar{\nu}}$ on $[t, \theta]$ and that the dynamics of $X_{t, x}^{\bar{\nu}}$ depends only on $\tilde{\nu}$ after $\theta$. Now observe that $\tilde{\nu}$ is independent of $\mathcal{F}_{s}$ and therefore on $\omega^{\theta(\omega)}$ since $\theta \leq s \mathbb{P}-$ a.s. It follows that

$$
\begin{aligned}
& \mathbb{E}\left[f\left(X_{t, x}^{\bar{\nu}}(T)\right) \mid \mathcal{F}_{\theta}\right](\omega)=\int f\left(X_{\theta(\omega), X_{t, x}^{\nu}(\theta)(\omega)}^{\tilde{\nu}\left(\mathbf{T}_{\theta(\omega)} \tilde{\omega}\right)}(T)\left(\mathbf{T}_{s} \tilde{\omega}\right)\right) d \mathbb{P}(\tilde{\omega}) \\
& =J\left(\theta(\omega), X_{t, x}^{\nu}(\theta)(\omega) ; \tilde{\nu}\right) \text {. }
\end{aligned}
$$

Remark 5.1 It can be similarly proved that A4' holds true, in the context of mixed controlstopping problems.

Let us now introduce the Hamiltonian of the control problem:

$$
H(t, x, p, A):=\inf _{u \in U} H^{u}(t, x, p, A), \quad(t, x, p, A) \in[0, T] \times \mathbb{R}^{d} \times \mathbb{R}^{d} \times \mathbb{M}^{d},
$$

where for $u \in U$ and $(t, x, p, A) \in \mathbf{S} \times \mathbb{R}^{d} \times \mathbb{M}^{d}$,

$$
H^{u}(t, x, p, A):=-\langle\mu(t, x, u), p\rangle-\frac{1}{2} \operatorname{Tr}\left[\left(\sigma \sigma^{\prime}\right)(t, x, u) A\right],
$$

and $\sigma^{\prime}$ is the transpose of the matrix $\sigma$. Notice that the operator $H$ is upper-semicontinuous. However, since the set $U$ may be unbounded, it may fail to be continuous. We therefore introduce the corresponding lower-semicontinuous envelope:

$$
H_{*}(z):=\liminf _{z^{\prime} \rightarrow z} H\left(z^{\prime}\right) \quad \text { for } \quad z=(t, x, p, A) \in \mathbf{S} \times \mathbb{R}^{d} \times \mathbb{M}^{d} .
$$

Since $V$ is locally bounded, the semicontinuous envelopes $V_{*}$ and $V^{*}$ are finite. 
Corollary 5.1 The following holds:

(i) $V^{*}$ is a viscosity subsolution of

$$
-\partial_{t} V^{*}+H_{*}\left(., D V^{*}, D^{2} V^{*}\right) \leq 0 \text { on }[0, T) \times \mathbb{R}^{d} .
$$

(ii) $\quad V_{*}$ is a viscosity supersolution of

$$
-\partial_{t} V_{*}+H\left(., D V_{*}, D^{2} V_{*}\right) \geq 0 \quad \text { on }[0, T) \times \mathbb{R}^{d} .
$$

Proof 1. We start with the supersolution property. Assume to the contrary that there is $\left(t_{0}, x_{0}\right) \in[0, T) \times \mathbb{R}^{d}$ together with a smooth function $\varphi:[0, T) \times \mathbb{R}^{d} \longrightarrow \mathbb{R}$ satisfying

$$
0=\left(V_{*}-\varphi\right)\left(t_{0}, x_{0}\right)<\left(V_{*}-\varphi\right)(t, x) \text { for all }(t, x) \in[0, T) \times \mathbb{R}^{d},(t, x) \neq\left(t_{0}, x_{0}\right),
$$

such that

$$
\left(-\partial_{t} \varphi+H\left(., D \varphi, D^{2} \varphi\right)\right)\left(t_{0}, x_{0}\right)<0 .
$$

Set

$$
\phi(t, x):=\varphi(t, x)-\left|t-t_{0}\right|^{2}-\left|x-x_{0}\right|^{4},
$$

and observe that $\left(\phi, \partial_{t} \phi, D \phi, D^{2} \phi\right)\left(t_{0}, x_{0}\right)=\left(\varphi, \partial_{t} \varphi, D \varphi, D^{2} \varphi\right)\left(t_{0}, x_{0}\right)$. In particular, (5.3) can be expressed in terms of $\phi$ :

$$
\left(-\partial_{t} \phi+H\left(., D \phi, D^{2} \phi\right)\right)\left(t_{0}, x_{0}\right)<0 .
$$

Then there exist $u \in U$ and $r>0$, with $t_{0}+r<T$, such that

$$
\left(-\partial_{t} \phi+H^{u}\left(., \phi, D \phi, D^{2} \phi\right)\right)(t, x)<0 \text { for all }(t, x) \in B_{r}\left(t_{0}, x_{0}\right) .
$$

Let $\left(t_{n}, x_{n}\right)_{n}$ be a sequence in $B_{r}\left(t_{0}, x_{0}\right)$ such that $\left(t_{n}, x_{n}, V\left(t_{n}, x_{n}\right)\right) \rightarrow\left(t_{0}, x_{0}, V_{*}\left(t_{0}, x_{0}\right)\right)$, and let $X^{n}:=X_{t_{n}, x_{n}}^{u}(\cdot)$ denote the solution of (5.1) with constant control $\nu=u$ and initial condition $X_{t_{n}}^{n}=x_{n}$, and consider the stopping time

$$
\theta_{n}:=\inf \left\{s \geq t_{n}:\left(s, X_{s}^{n}\right) \notin B_{r}\left(t_{0}, x_{0}\right)\right\} .
$$

Applying Itô's formula to $\phi\left(\cdot, X^{n}\right)$, and using (5.4) and (5.2), we see that

$$
\phi\left(t_{n}, x_{n}\right)=\mathbb{E}\left[\phi\left(\theta_{n}, X_{\theta_{n}}^{n}\right)-\int_{t_{n}}^{\theta_{n}}\left[\partial_{t} \phi-H^{u}\left(., D \phi, D^{2} \phi\right)\right]\left(s, X_{s}^{n}\right) d s\right] \leq \mathbb{E}\left[\phi\left(\theta_{n}, X_{\theta_{n}}^{n}\right)\right] .
$$

Now observe that $\varphi \geq \phi+2 \eta$ on $\partial B_{r}\left(t_{0}, x_{0}\right)$ for some $\eta>0$. Hence, the above inequality implies that $\phi\left(t_{n}, x_{n}\right) \leq \mathbb{E}\left[\varphi\left(\theta_{n}, X_{\theta_{n}}^{n}\right)\right]-\eta$. Since $(\phi-V)\left(t_{n}, x_{n}\right) \rightarrow 0$, we can then find $n$ large enough so that

$$
V\left(t_{n}, x_{n}\right) \leq \mathbb{E}\left[\varphi\left(\theta_{n}, X_{\theta_{n}}^{n}\right)\right]-\eta \text { for sufficiently large } n \geq 1 .
$$


On the other hand, it follows from (3.2) that:

$$
V\left(t_{n}, x_{n}\right) \geq \sup _{\nu \in \mathcal{U}_{t_{n}}} \mathbb{E}\left[\varphi\left(\theta_{n}, X_{t_{n}, x_{n}}^{\nu}\left(\theta_{n}\right)\right)\right] \geq \mathbb{E}\left[\varphi\left(\theta_{n}, X_{\theta_{n}}^{n}\right)\right]
$$

which is the required contradiction.

2. We now prove the subsolution property. Assume to the contrary that there is $\left(t_{0}, x_{0}\right) \in$ $[0, T) \times \mathbb{R}^{d}$ together with a smooth function $\varphi:[0, T) \times \mathbb{R}^{d} \longrightarrow \mathbb{R}$ satisfying

$$
0=\left(V^{*}-\varphi\right)\left(t_{0}, x_{0}\right)>\left(V^{*}-\varphi\right)(t, x) \text { for all }(t, x) \in[0, T) \times \mathbb{R}^{d},(t, x) \neq\left(t_{0}, x_{0}\right),
$$

such that

$$
\left(-\partial_{t} \varphi+H_{*}\left(., D \varphi, D^{2} \varphi\right)\right)\left(t_{0}, x_{0}\right)>0 .
$$

We can then find $r>0$ such that $t_{0}+r<T$ and

$$
\left(-\partial_{t} \varphi+H^{u}\left(., D \varphi, D^{2} \varphi\right)\right)(t, x)>0 \quad \text { for every } u \in U \text { and }(t, x) \in B_{r}\left(t_{0}, x_{0}\right) .
$$

Since $\left(t_{0}, x_{0}\right)$ is a strict maximizer of the difference $V^{*}-\varphi$, it follows that

$$
-2 \eta:=\max _{\partial B_{r}\left(t_{0}, x_{0}\right)}\left(V^{*}-\varphi\right)<0
$$

Let $\left(t_{n}, x_{n}\right)_{n}$ be a sequence in $B_{r}\left(t_{0}, x_{0}\right)$ such that $\left(t_{n}, x_{n}, V\left(t_{n}, x_{n}\right)\right) \rightarrow\left(t_{0}, x_{0}, V^{*}\left(t_{0}, x_{0}\right)\right)$. For an arbitrary control $\nu^{n} \in \mathcal{U}_{t_{n}}$, let $X^{n}:=X_{t_{n}, x_{n}}^{\nu^{n}}$ denote the solution of (5.1) with initial condition $X_{t_{n}}^{n}=x_{n}$, and set

$$
\theta_{n}:=\inf \left\{s \geq t_{n}:\left(s, X_{s}^{n}\right) \notin B_{r}\left(t_{0}, x_{0}\right)\right\}
$$

We may assume without loss of generality that

$$
\left|(V-\varphi)\left(t_{n}, x_{n}\right)\right| \leq \eta \text { for all } n \geq 1
$$

Applying Itô's formula to $\varphi\left(\cdot, X^{n}\right)$ and using (5.6) together with (5.2) leads to

$$
\varphi\left(t_{n}, x_{n}\right)=\mathbb{E}\left[\varphi\left(\theta_{n}, X_{\theta_{n}}^{n}\right)-\int_{t_{n}}^{\theta_{n}}\left[\partial_{t} \varphi-H^{\nu^{n}}\left(., D \varphi, D^{2} \varphi\right)\right]\left(s, X_{s}^{n}\right) d s\right] \geq \mathbb{E}\left[\varphi\left(\theta_{n}, X_{\theta_{n}}^{n}\right)\right] .
$$

Now observe that $\varphi \geq V^{*}+2 \eta$ on $\partial B_{r}\left(t_{0}, x_{0}\right)$ by (5.7). Hence, the above inequality implies that $\varphi\left(t_{n}, x_{n}\right) \geq \mathbb{E}\left[V^{*}\left(\theta_{n}, X_{\theta_{n}}^{n}\right)\right]+2 \eta$, which implies by (5.8) that:

$$
V\left(t_{n}, x_{n}\right) \geq \mathbb{E}\left[V^{*}\left(\theta_{n}, X_{\theta_{n}}^{n}\right)\right]+\eta \text { for } n \geq 1
$$

Since $\nu^{n} \in \mathcal{U}_{t_{n}}$ is arbitrary, this contradicts (3.1) for $n \geq 1$ fixed. 


\section{References}

[1] Bertsekas D.P. and S.E. Shreve (1978), Stochastic Optimal Control : The Discrete Time Case, Mathematics in Science and Engineering 139, Academic Press.

[2] Borkar V.S. (1989), Optimal Control of Diffusion Processes, Pitman Research Notes 203. Longman Sci. and Tech. Harlow, UK.

[3] Crandall M.G., H. Ishii and P.-L. Lions (1992), User's guide to viscosity solutions of second order Partial Differential Equations, Amer. Math. Soc. 27, 1-67.

[4] El Karoui N. (1981), Les Aspects probabilistes du contrôle stochastique, Springer Lecture Notes in Mathematics 876, Springer Verlag, New York.

[5] Fleming W.H. and H.M. Soner (2006), Controlled Markov Processes and Viscosity Solutions, Second Edition, Springer.

[6] Lions P.-L. (1983), Optimal Control of Diffusion Processes and Hamilton-Jacobi-Bellman Equations I, Comm. PDE. 8, 1101-1134.

[7] Lions P.-L. (1983), Optimal Control of Diffusion Processes and Hamilton-Jacobi-Bellman Equations, Part II: Viscosity Solutions and Uniqueness, Comm. PDE. 8, 1101-1134.

[8] Reny P. J. (1999), On the Existence of Pure and Mixed Strategy Nash Equilibria in Discontinuous Games, Econometrica, 67(5), 1029-1056.

[9] Touzi N. (2002), Stochastic Control Problems, Viscosity Solutions, and Application to Finance, Quaderni, Edizioni della Scuola Normale Superiore, Pisa. 